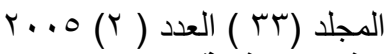 \\ مجلة زر اعة الر افدين
}

تأثير النسجة والكين الكاربونات الكلية في لدانة التربة لبعض المواقع في محافظة نينوى العدى

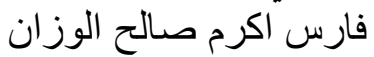

قسم علوم التربة و المياه / كلية الزراعة والغابات/ جامعة الموصل/العراق

\section{الخلاصة}

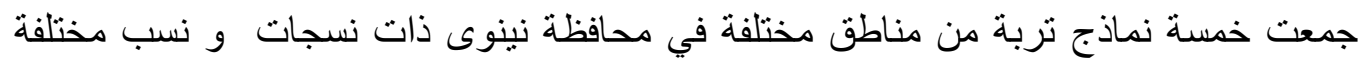

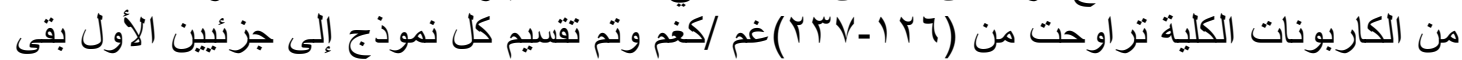

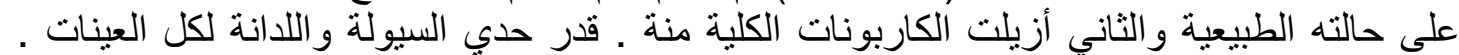

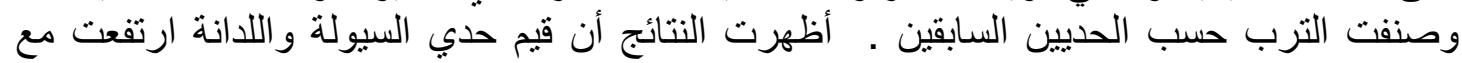

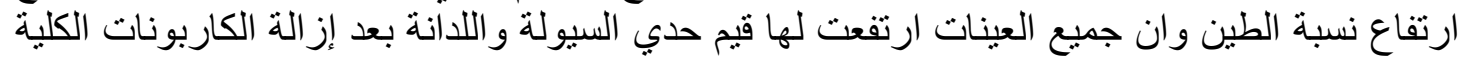

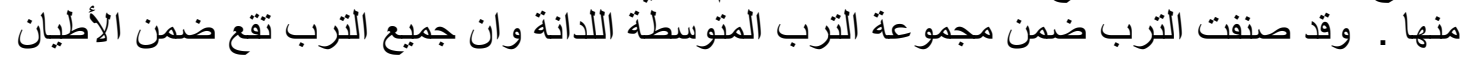

\section{المقدمة}

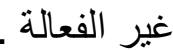

يعد دليل اللدانة قياسا غير مباشر لتكوين أشكال من التربة وهو يمثل دالة لعدد الأغلفة المائية

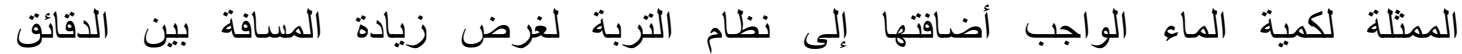

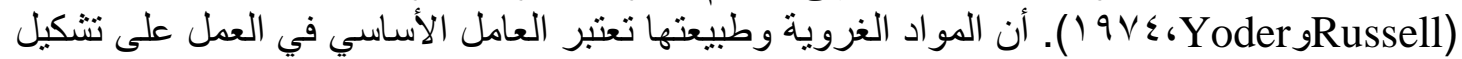

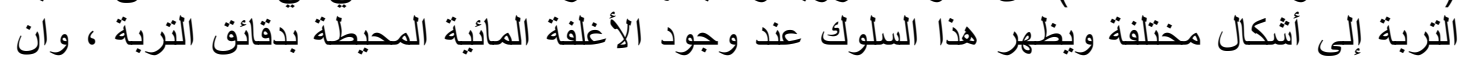

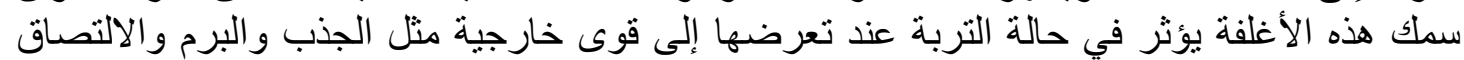

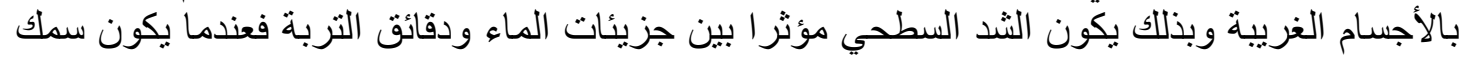

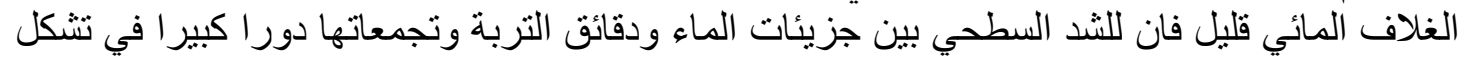

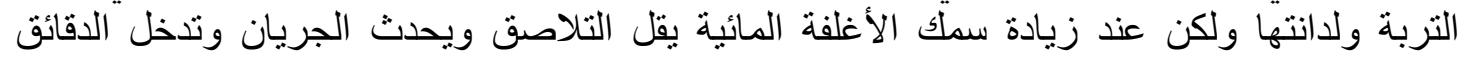

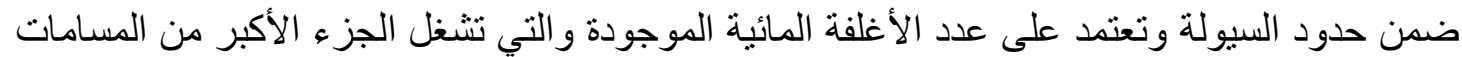

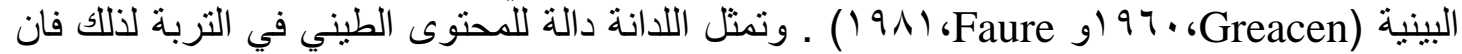

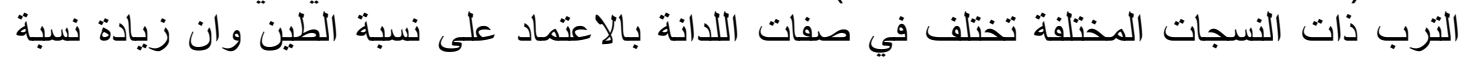

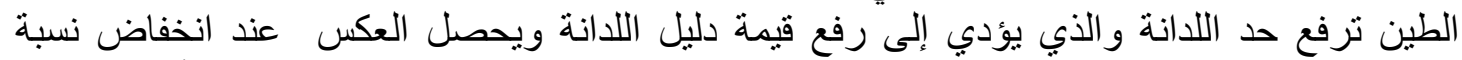

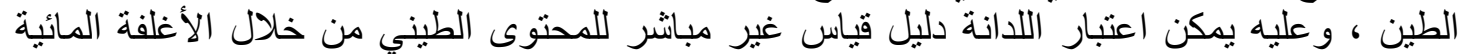

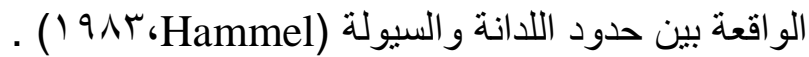

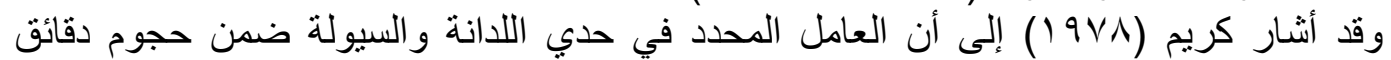

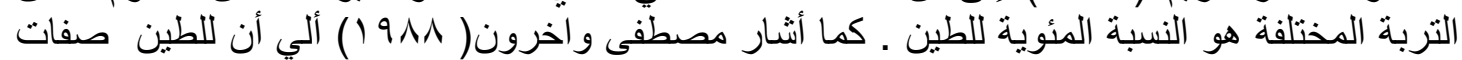

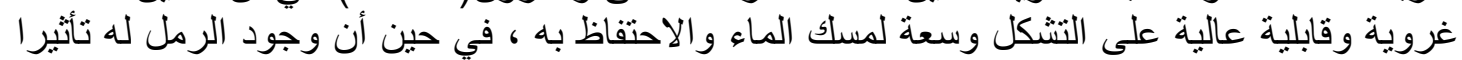

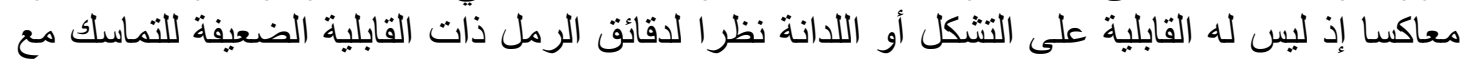

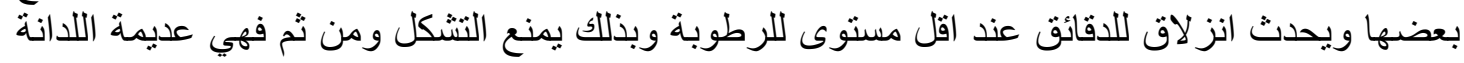

إضافة إلى ما سبق فان للكاربونات الكلية ارتباط معنوي مع فعالية الطين حيث ترتفع قيم فعالية

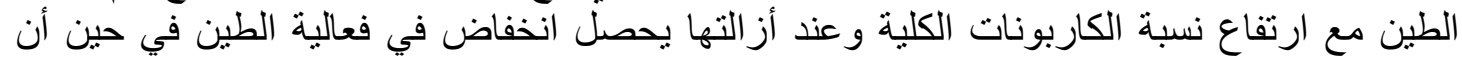

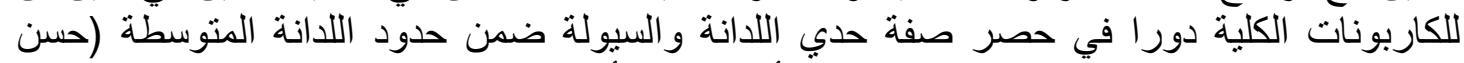

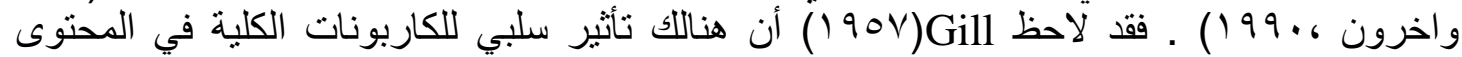

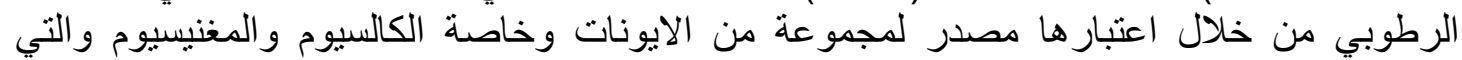

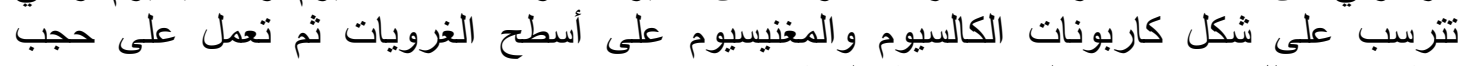

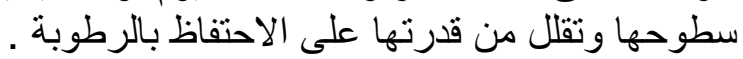

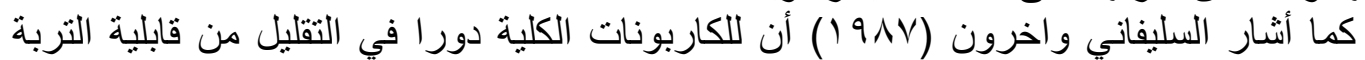

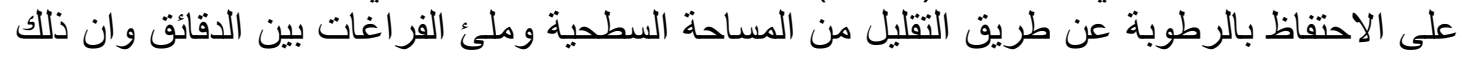

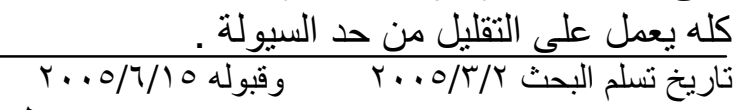




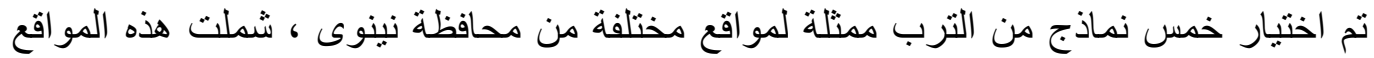

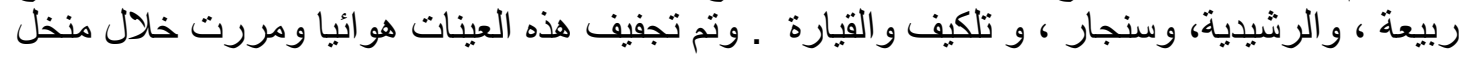

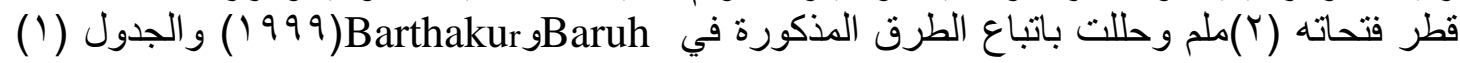

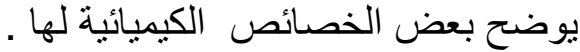

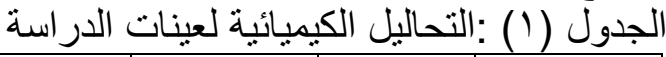

\begin{tabular}{|c|c|c|c|c|c|c|}
\hline 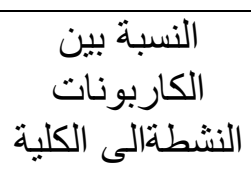 & الكاربونات النشطة & الكاربونات & غم العضوية & $\begin{array}{c}\text { Ec } \\
\text { سيمنز/2 }\end{array}$ & $\mathrm{pH}$ & الموقع \\
\hline. & 0 . & $1 Y 7$ & r. 11. & 1.0 & V.rY & ربيعة \\
\hline$\cdot r v I$ & 10 & rrq & 1.90 & .0 & $V .01$ & الرشيدية \\
\hline$\because$ rAr & 9. & rTo & $1 . . \wedge$. & r.A & V.r. & سنجار \\
\hline$\because \varepsilon \ldots$ & 90 & rTV & $11 . \leqslant 0$ & 1.7 & $V . Y Y$ & تلكيف \\
\hline . ro. & $V$. & r.. & 9.10 & $\because V$ & $V .0$. & القيارة \\
\hline
\end{tabular}

ومن اجل ملاحظة تأثثر الكاربونات الكلية فقد قسمت كل عينة إلى قسمين الأول بقى على حالته

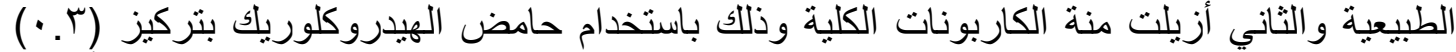

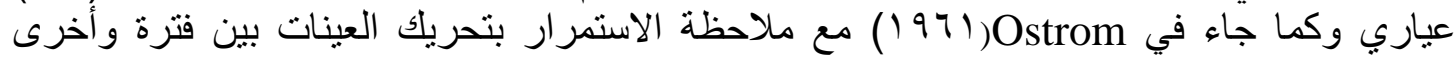

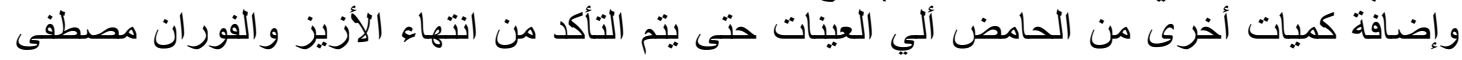

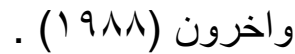

وتم تقدير حد السيولة لجميع عينات التربة قبل وبعد إزالة الكاربونات الكلية باستخدام جهاز وكما جاء في (Atterberg device)

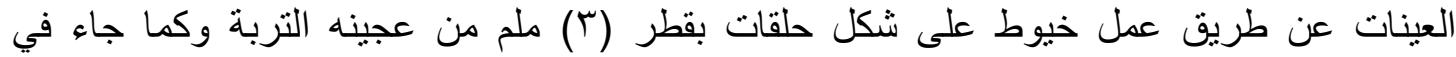
أما دليل اللانة فتم حسابه من خلاهل الفرف بين المحتوى الرطوبي عند حدي اللانة الأعلى و الأدنى

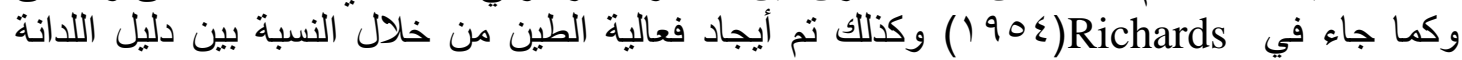

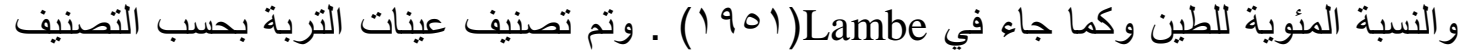
المقترح من قبل

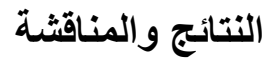

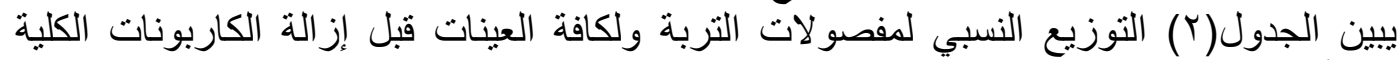

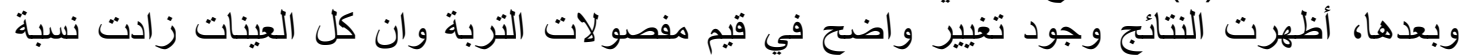

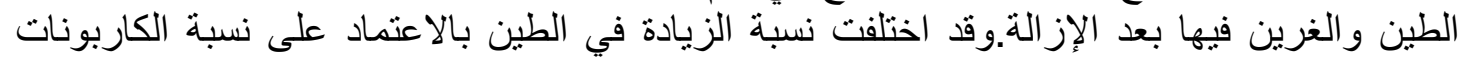

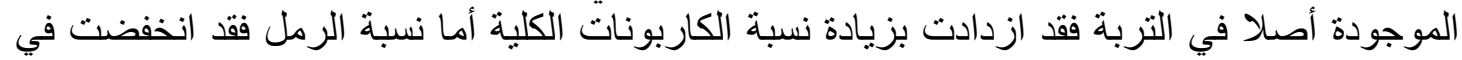

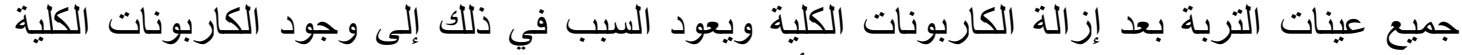

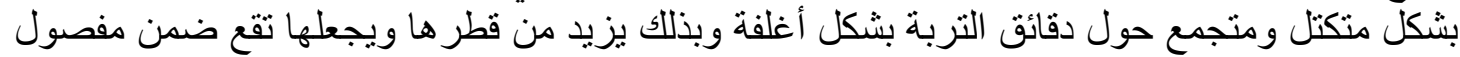

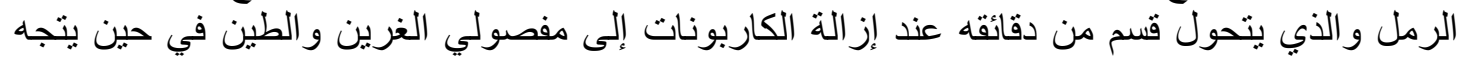

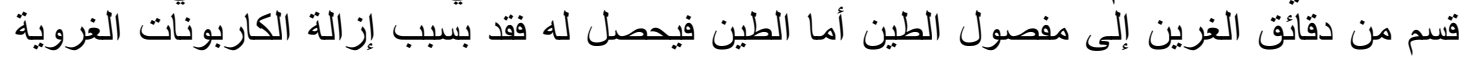

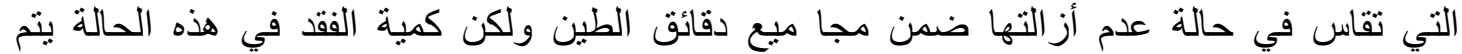

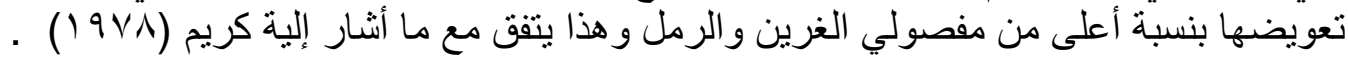

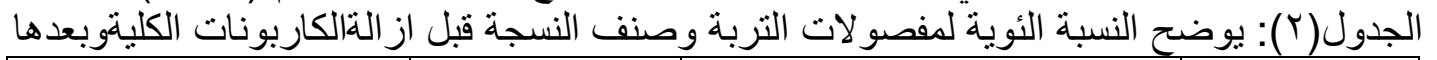




\begin{tabular}{|c|c|c|c|c|c|}
\hline & ميكاغر ام/مج & 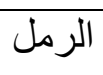 & الغرين & الطين & \\
\hline طينية & 1.199 & 19 & $r q$ & $\varepsilon r$ & ربيعة A \\
\hline طينية غرينية & --- & 10 & \&1 & $\varepsilon \varepsilon$ & ر ربيعة B ر \\
\hline مزيجية طينية & $1 . Y .$. & ro & $\varepsilon r$ & r & A الرشيدية A \\
\hline مزيجية طينية غرينية & --- & 11 & $\leqslant 0$ & rv & الرشيدية B \\
\hline مزيجية طينية رملية & $1 . r \leq 0$ & $\varepsilon 9$ & 11 & Tr & A سنجار A \\
\hline مزيجية طبنية & --- & $\varepsilon$. & YI & rq & B سنجار B \\
\hline مزيجية & $1.1 Y V$ & Ts & $\varepsilon 1$ & To & A تلكيف A \\
\hline مزيجية طينية & --- & rT & $\varepsilon r$ & $\mu_{1}$ & تلكيف B \\
\hline مزيجية طينية غرينية & $1 . Y M$ & IV & $\leqslant 0$ & ऍᄉ & A القيارة A \\
\hline طينية غرينية & --- & $1 \%$ & $\sum V$ & $\sum 1$ & القيارة B \\
\hline
\end{tabular}

لذلك سوف يكون هنالك ارتباط بين التوزيع النسبي لمفصولات التربة (النسجة) وبين

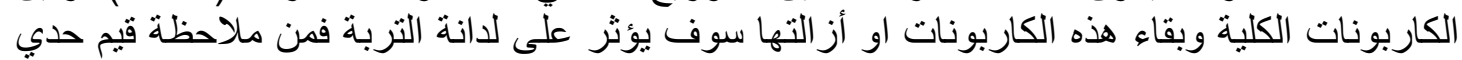

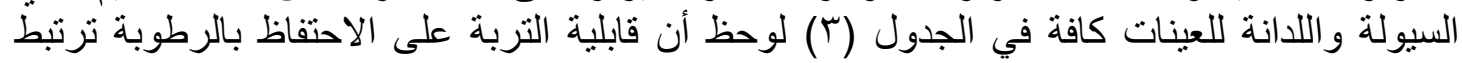

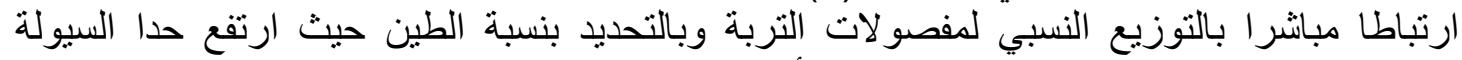

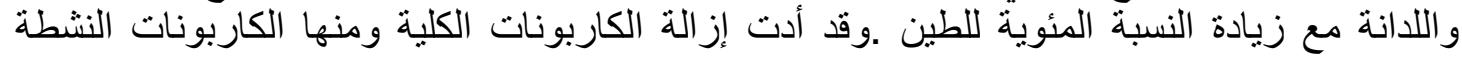

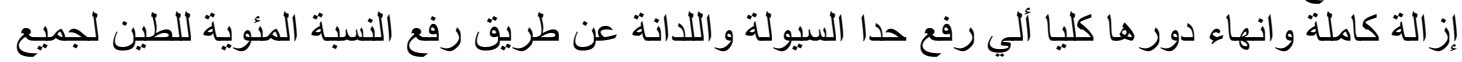

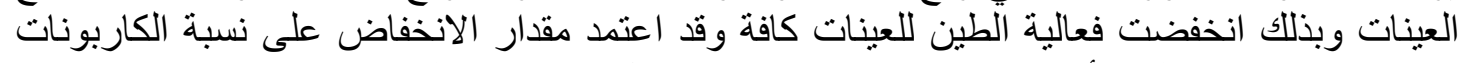

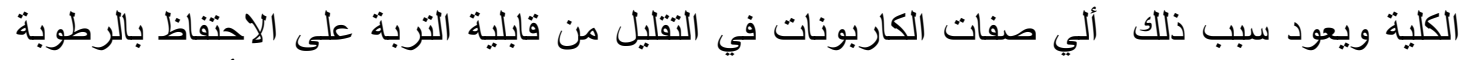

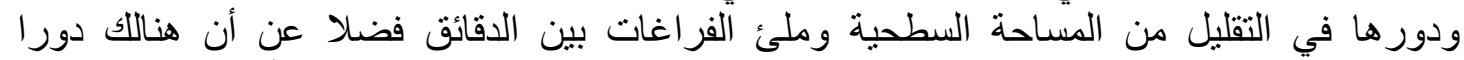

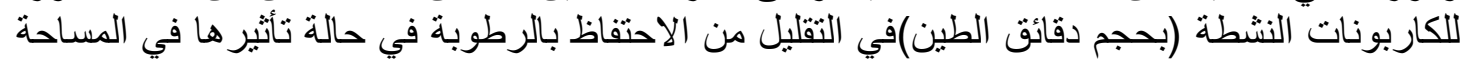

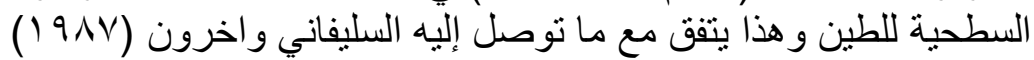

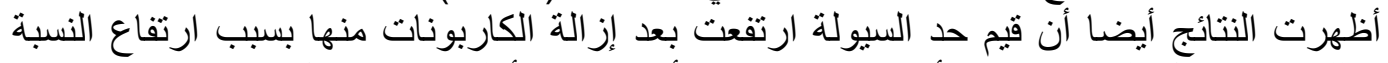

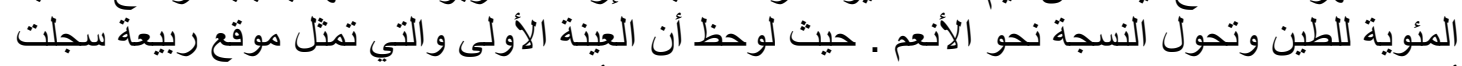

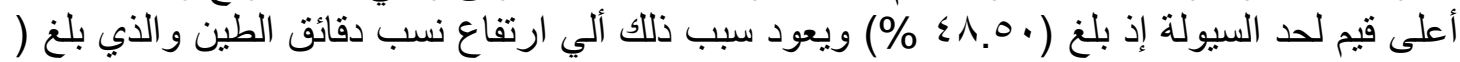

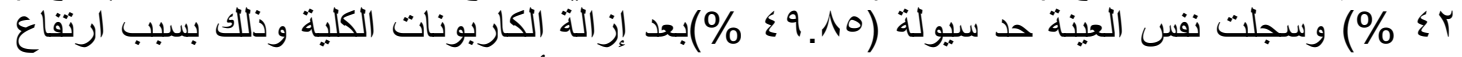

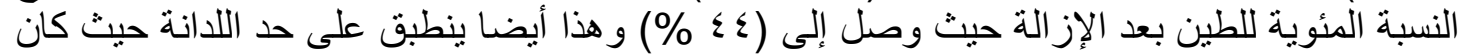

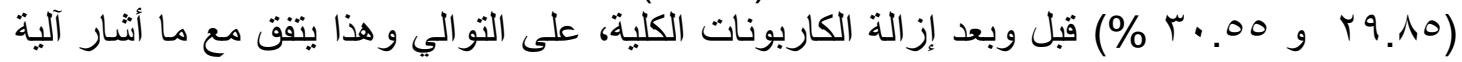

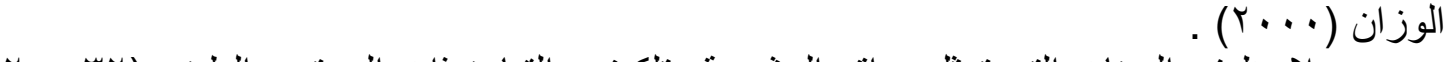

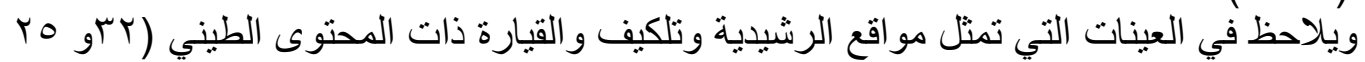

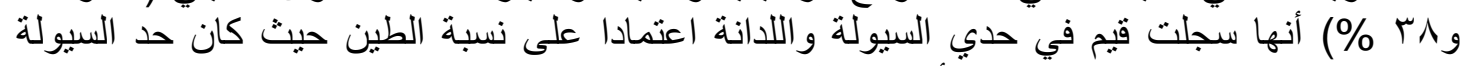

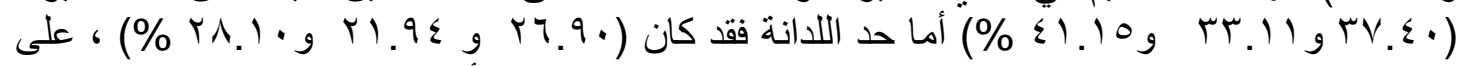

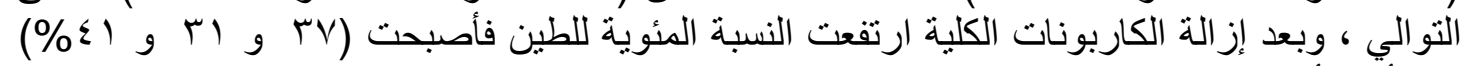

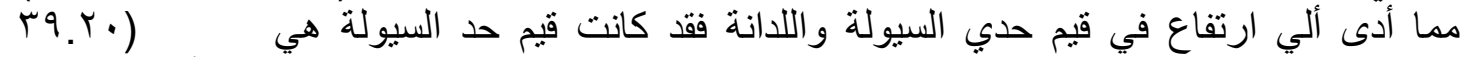

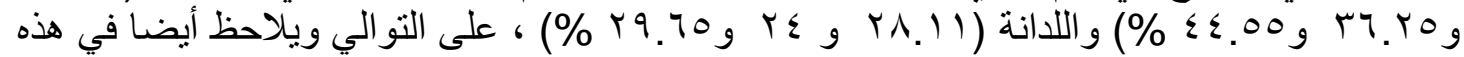

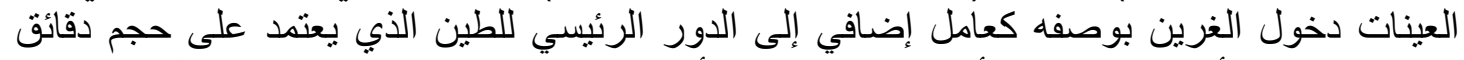

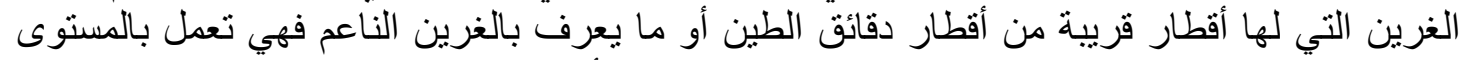

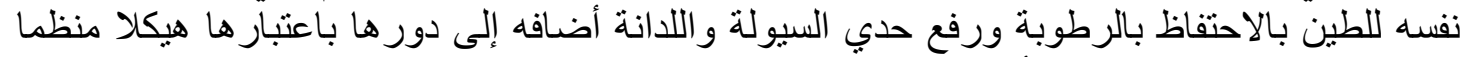

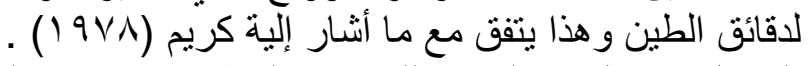

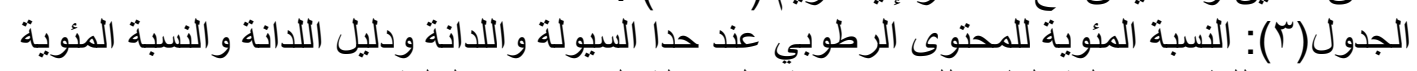

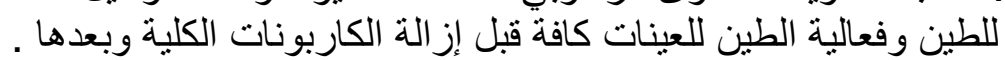

\begin{tabular}{|c|c|c|c|}
\hline 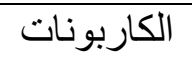 & 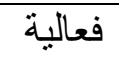 & النسبة المئوية للمحتوى الرطوبي عند & النسبة الئوية \\
\hline
\end{tabular}




\begin{tabular}{|c|c|c|c|c|c|c|}
\hline الكلية غم/كغم & الطين & دليل اللدانة & حد اللدانة & حد السيولة & للطين & \\
\hline $1 \times 7$ & $\because \leq \leqslant \leq$ & 11.70 & 19.10 & $\leqslant \wedge .0$ & $\varepsilon Y$ & ربيعة A \\
\hline--- &.$\leqslant \mu$ & 19.5. & $r .00$ & $\leqslant 9 . \wedge$. & $\varepsilon \varepsilon$ & ربيعة B \\
\hline rrq &. MrA & 1.00 & r..9. & $r v . \varepsilon$. & Tr & Aالرشيدية \\
\hline --- & .199 & 11.99 & rA. 11 & rq.r. & rV & B B B Bيدية \\
\hline Tro & $\cdot r r q$ & $1 \cdot . \wedge 1$ & $r \cdot . r \cdot$ & 1.11 & Tr & Aنجار A \\
\hline --- & $\cdot r \cdot r$ & 11.11 & r.M & $r \varepsilon .1 T$ & rq & سنجار B \\
\hline rrv & $\because \leqslant \leqslant 7$ & $11.1 \mathrm{~V}$ & Y). $9 \varepsilon$ & r.11 & ro & تلكيف A \\
\hline--- & .190 & IY.YO & $r \leqslant .$. & r. Yo & rI & تلكيف B \\
\hline$r \ldots$ & . & $1 \pi .10$ & r^.1. & $\leqslant 1.90$ & rᄉ & A القيارة A \\
\hline--- & זוצ. & $1 \leq .9$. & r9.70 & $\leqslant \leqslant .00$ & $\leqslant 1$ & القيارة B \\
\hline
\end{tabular}

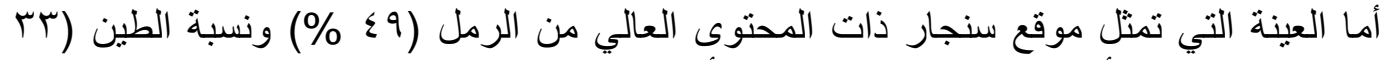

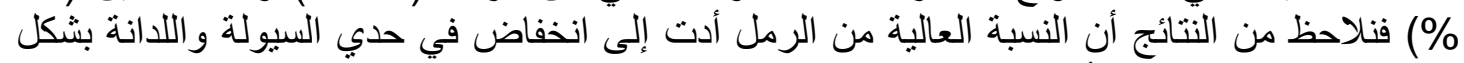

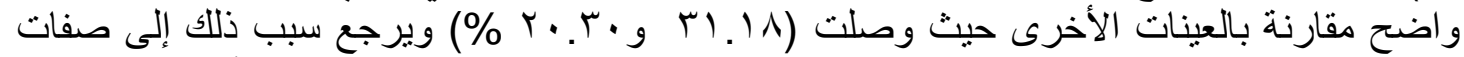

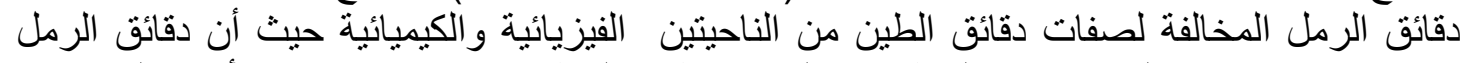

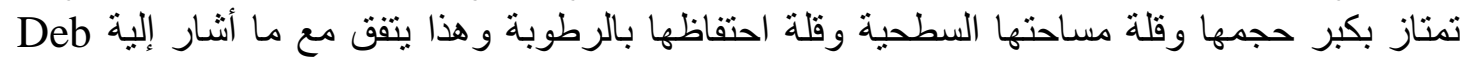
( $19 \vee 9$ ) Chadha

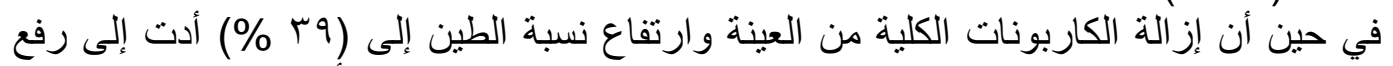

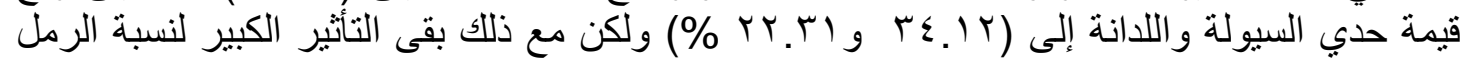

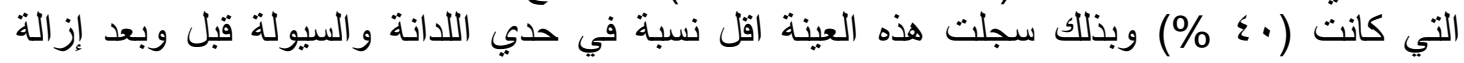
الكاربونات الكلية .

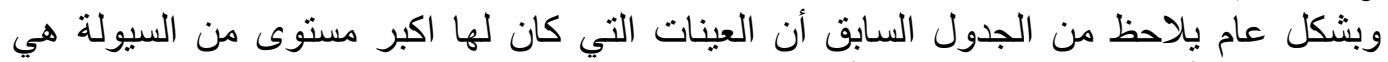

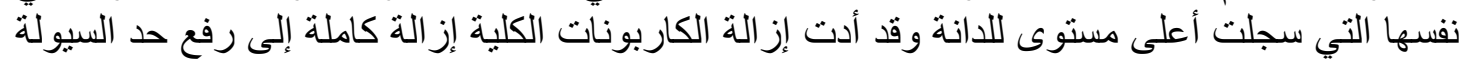

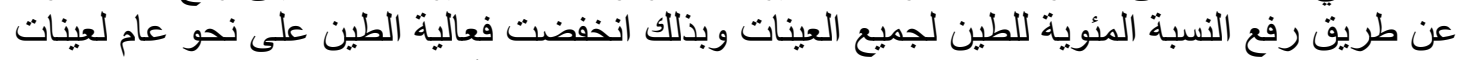

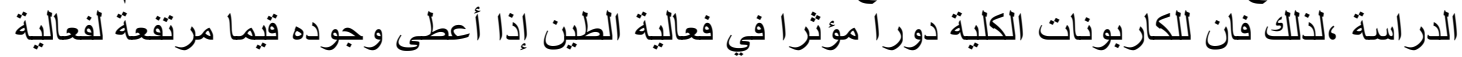

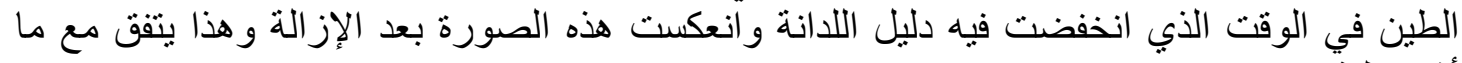
أشنار إلية حسن واخرون (199 (19) )

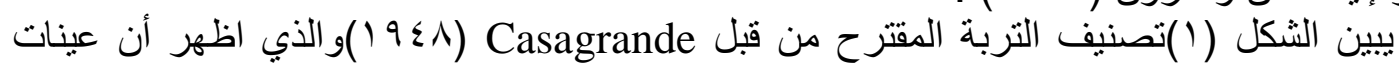

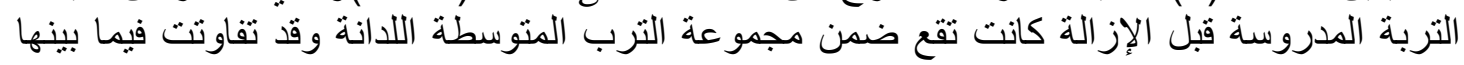

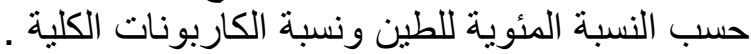

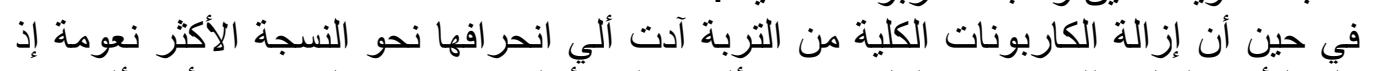

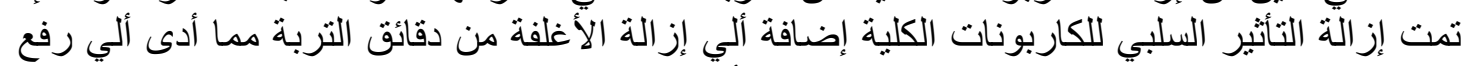

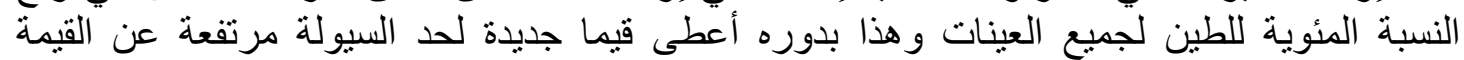

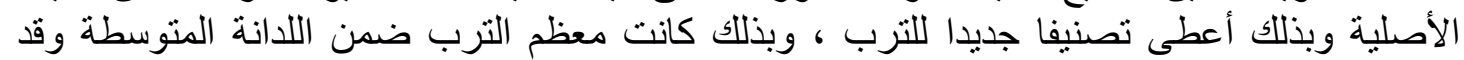

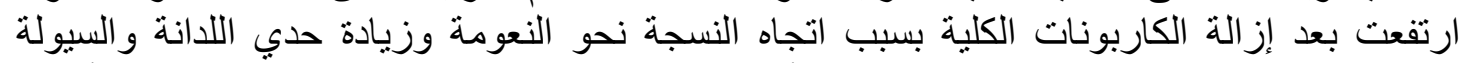

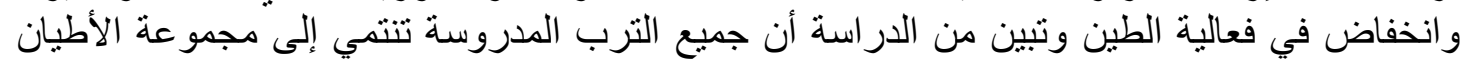

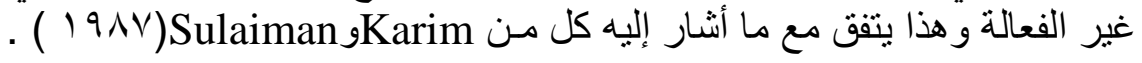




\title{
EFFECT OF THE SOILTEXTURE AND TOTAL CARBONATE IN SOIL CONSISTENCY FOR SOME LOCATIONS IN NINAVAH
}

\author{
Faris Akram Saleh AI-Wazan
}

Soil and Water Sci , Dept., College of Agric. and Foresty, Mosul Univ.,Iraq

\begin{abstract}
Five soil samples collected from different locations in Nineva province with different texture and total carbonate content $(126-237 \mathrm{gm} / \mathrm{Kg})$. Each soil sample was divided in to two parts the first one was kept on its natural condition however the total carbonate was removed from the second one .The studied upper and lower limits and classification of soil according to casagrande (1948). Indicated that the results both liquid and plastic limits increased as clay content increased . and removed of total carbonate showed that liquid and plastic limits are increased compared with natural soil samples . Medium plasticity group according to casagrande are classified the result showed that all the soil belonged to inactive clays .

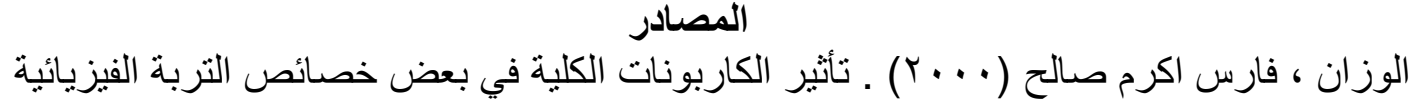

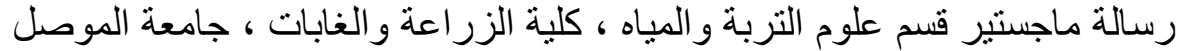

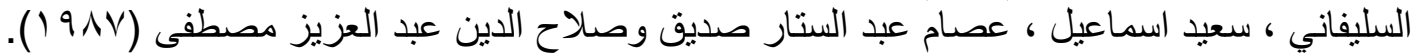

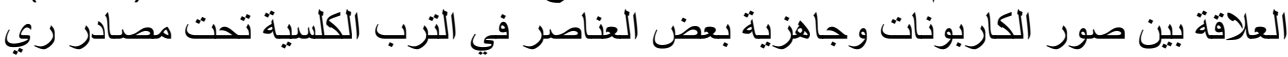

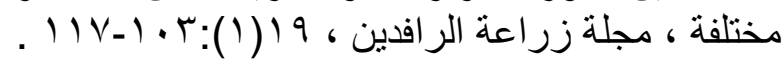

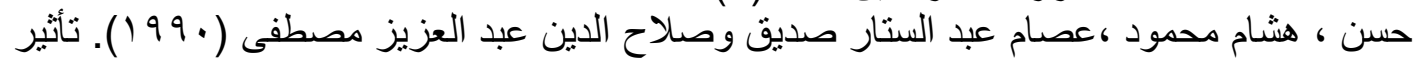

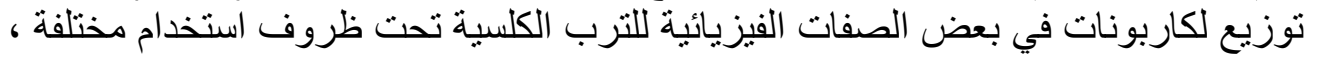

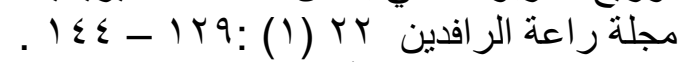

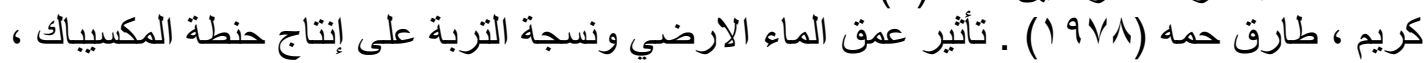

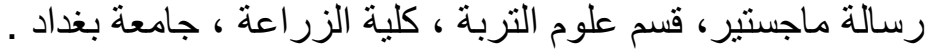

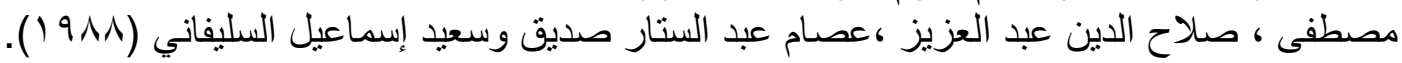

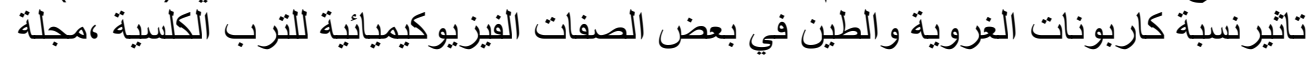

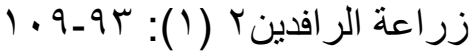

Baruh , T.C. and H.P. Barthakur (1999). A text Book of Soil analysis

Casagrande,A. (1948) Classification and Identification of Soils In :Lambe,

T.W.(1951). Soil Testing for Engineers . TASCE.1(3),: 901

Deb , B.C. and S.P. Chadha (1979).Mechanical analysis of calcareous soils and istribution of calcium carbonate in various fractions .Soil. Sci. Soc. J. Indian 18 ( 3): 227-232

Faure, A.(1981).Anew conception of the plastic and limits of clay . Soil.Tillage es., 1 (1):97-105 .

Gill , W.R.andC.A. Reaves (1957).Relation ship of atterberg limits to some other Properties of illinois soils .Soil.Sci.Soc.J.Am. 21 ( 2):491-494. 
Greacen ,E.L.(1960).Aggregate strength and soil consistence .Trans.Int.Congr of soil Sci., (1):256-264 .

Hammel , J.E., M.E. Summer and J. Burema (1983).Atterberg limits as indices of External surface areas of soil. Soil. Sci. Soc. J.Am. 47 (.3 ): 1054 1056

Karim ,T.H.and M.S. Sulaiman (1987).Changes in some physical properties of some Calcareous soil in the north part of iraq as affected by decalcification .Iraq . J.Agr.Sci.(Zanco). 5 (.3) :83-94 .

Lambe ,T.W.(1951).Soil tests for engineers wiley eastern limited .New Delhi Ostrom ,M.E. (1961).Separation of clay minerals from carbonate rocks by using acid

Sediment petrol .B1 P:123-129 .

Richards ,L.A. (1954)Diagnosis and improvement of saline and alkaline soils Agriculture hand book No. 60, USDA.

Russell ,M.B.and A.R.Yoder (1974). Determination of atterberg limits using moisture

Tension methods .Trans. Res., 49(1):69-80. 